\title{
Identification of Prognostic Gene Signature Associated with Tumor Microenvironment of Cholangiocarcinoma
}

\section{Jixiang Cao}

Peking University Health Science Centre https://orcid.org/0000-0002-2224-4407

\section{Xi Chen}

Peking University Third Hospital

\section{Guang Lu}

National University of Singapore

\section{Haowei Wang}

Peking University Health Science Centre

\section{Xinyu Zhang}

Peking University Health Science Centre

\section{Hua Yang}

Peking University Health Science Centre

\section{Yun Bai ( $\boldsymbol{Q}$ baiyun@bjmu.edu.cn )}

Peking University Health Science Center https://orcid.org/0000-0002-2688-9229

\section{Primary research}

Keywords: Tumor Immune Estimation Resource (TIMER), Cholangiocarcinoma (CCA), tumor microenvironment (TME), CCA

Posted Date: May 21st, 2021

DOI: https://doi.org/10.21203/rs.3.rs-524410/v1

License: (a) (1) This work is licensed under a Creative Commons Attribution 4.0 International License. Read Full License 


\title{
Identification of Prognostic Gene Signature Associated with Tumor Microenvironment of Cholangiocarcinoma
}

\author{
Jixiang Cao ${ }^{1}$, Xi Chen ${ }^{2}$, Guang $\mathrm{Lu}^{3}$, Haowei Wang1, Xinyu Zhang1, Hua Yang ${ }^{*}$, \\ Yun Bai ${ }^{1 *}$ \\ ${ }^{1}$ Department of Cell Biology, Peking University Health Science Center, Xue Yuan \\ Road 38, Beijing, China \\ ${ }^{2}$ Department of Cardiology, Peking University Third Hospital, North garden Road 49, \\ Haidian District, Beijing, China \\ ${ }^{3}$ Department of Physiology, Yong Loo Lin School of Medicine, National University of \\ Singapore, Singapore, Singapore \\ * Correspondence: \\ Hua Yang \\ yanghua@bjmu.edu.cn \\ Yun Bai \\ baiyun@bjmu.edu.cn
}

Keywords: Cholangiocarcinoma, TCGA, Tumor microenvironment, TMErelated DEGs, prognostic model, Tumor infiltrating immune cells.

\begin{abstract}
Background: Cholangiocarcinoma (CCA) is the most common malignancy of the biliary tract with a dismal prognosis. Increasing evidence suggests that tumor microenvironment (TME) is closely associated with cancer prognosis. However, the prognostic signature for CCA based on TME has not yet been reported. This study aimed to develop a TME-related prognostic signature for accurately predicting the prognosis of patients with CCA.
\end{abstract}

Methods: Based on the TCGA database, we calculated the stromal and immune scores using the ESTIMATE algorithm to assess TME in stromal and immune cells derived from CCA. TME-related differentially expressed genes were identified, followed by functional enrichment analysis and PPI network analysis. Univariate Cox regression analysis, Lasso Cox regression model and multivariable Cox regression analysis were performed to identify and construct the TME-related prognostic gene signature. Gene Set Enrichment Analyses (GSEA) was performed to further investigate the potential molecular mechanisms. The correlations between the risk scores and tumor infiltration 
immune cells were analyzed using Tumor Immune Estimation Resource (TIMER) database.

Results: A total of 784 TME-related differentially expressed genes (DEGs) were identified, which were mainly enriched in immune-related processes and pathways. Among these TME-related DEGs, A novel two-gene signature (including GAD1 and KLRB1) was constructed for CCA prognosis prediction. The AUC of the prognostic model for predicting the survival of patients at 1-, 2-, and 3- years was $0.811,0.772$, and 0.844 , respectively. Cox regression analysis showed that the two-gene signature was an independent prognostic factor. Based on the risk scores of the prognostic model, CCA patients were divided into high- and low-risk groups, and patients with high-risk score had shorter survival time than those with low-risk score. Furthermore, we found that the risk scores were negatively correlated with TME-scores and the number of several tumor infiltration immune cells, including B cells and CD4+ T cells.

Conclusion: Our study established a novel TME-related gene signature to predict the prognosis of patients with CCA. This might provide a new understanding of the potential relationship between TME and CCA prognosis, and serve as a prognosis stratification tool for guiding personalized treatment of CCA patients.

\section{Background}

Cholangiocarcinoma (CCA) is one of the most common malignancies of the biliary tract and also the second most common hepatic malignancy after hepatocellular carcinoma. Based on the anatomic location, CCA can be classified into 3 subtypes : intrahepatic (iCCA), perihilar (pCCA), and distal (dCCA) subtypes [1]. Despite some therapeutic progress has been achieved, the prognosis of CCA remains dismal [2]. Clinicopathological parameters such as the TNM stage, completeness of the procedure (R0/R1) and lymph node metastases have been used to predict the prognosis of CCA patients [3, 4]. However, these prognostic markers have limitations in the prediction of prognosis of CCA patients. Thus, more reliable prognostic markers are needed to improve such assessment.

Tumor microenvironment (TME) has been reported to play pivotal roles in tumor development, progression and recurrence [5]. Increasing evidence shows that TME is closely associated with cancer prognosis [6-8]. TME consists of surrounding cells and noncellular components. Tumor-infiltrating stromal cells and immune cells as the major cell components of surrounding cells that play a significant role in cancer prognosis [911]. For example, Vigano et al. found that tumor-infiltrating lymphocytes and macrophages were associated with prognosis in intrahepatic cholangiocellular carcinoma patients after complete surgery [12]. To access the fraction of stromal and immune cells in tumor samples, Estimation of STromal and Immune cells in Malignant Tumors using Expression data (ESTIMATE) has been developed as an open online tool [13]. Accumulating evidence has indicated that tumor-infiltrating stromal cells and immune cells were correlated with prognosis in patients with several tumors using 
ESTIMATE algorithm. However, these prognostic factors have not been investigated in CCA. Thus, using tumor-infiltrating stromal cells and immune cells to predict CCA patient's prognosis still needs further investigation.

Considering the prognostic potential of TME in CCA, we calculated the stromal and immune scores of CCA patients with the ESTIMATE algorithm in the current study and established a novel TME-related prognostic model for CCA. Our results may serve as a prognosis stratification tool for guiding personalized treatment of CCA patients.

\section{Materials and methods}

\section{Data collection and calculation of stromal and immune scores}

We download transcriptome RNA-sequencing data and clinical data of cholangiocarcinoma patients from The Cancer Genome Atlas (TCGA) database, and a total of 32 patients were selected according to the screening criteria. The clinical information of CCA patients is shown in Table 1. The stromal and immune scores were calculated through the ESTIMATE algorithm using the "estimate" package in R. All patients were divided into high- and low- stromal/immune-score groups based on the median stromal/immune scores.

\section{Differential expression analysis}

Differentially expressed genes (DEGs) between high- and low- stromal/immune-score groups were identified using "DESeq2" package in R software. $\mid \log 2$ fold-change $\mid>1$ and adjusted $\mathrm{P}$ value (FDR) $<0.05$ was set as the threshold for DEG identification.

\section{Function enrichment analysis and protein-protein interaction (PPI) network construction}

Functional enrichment analysis was carried out using "clusterProfiler" package in R, including Gene Ontology (GO) and Kyoto Encyclopedia of Genes and Genomes (KEGG). GO terms are composed of biological process (BP), cellular component (CC), and molecular function (MF). Adjusted P-value $<0.05$ was considered statistically significant.

The protein-protein interaction (PPI) network was constructed using the Search Tool for the Retrieval of Interacting Genes (STRING) database [14]. Cytoscape software (version: 3.6.0) was utilized to visualize the molecular interaction networks [15]. The top 3 modules of PPI network were selected using the Molecular Complex Detection (MCODE), which is a plugin in Cytoscape. The degree cut-off $=2$, node score cut-off $=0.2$, $\mathrm{k}$-core $=2$, and maxinum depth $=100$ were regarded as standard.

\section{Construction of TME-related prognostic model}

Univariate Cox regression analysis was performed to identify survival-associated DEGs according to the threshold value of $p<0.05$ using the "survival" package in $R$. 
Then, we conducted a least absolute shrinkage and selection operator (LASSO) and multivariable Cox regression analysis to identify TME-related prognostic genes using the "glmnet" and "survival" package, respectively in R. The calculation formula of risk score for CCA patients was shown as follows:

Risk score $=$ Coef $1 * \operatorname{Exp} 1+$ Coef $2 * \operatorname{Exp} 2+$ Coefi $*$ Expi,

Coefi was the regression coefficient which was calculated by multivariate Cox regression model, and Expi was the gene expression level. Patients were divided into a high- and low-risk groups based on the median Risk score.

\section{Gene set enrichment analysis (GSEA)}

To investigate the differences of the potential molecular mechanisms of the TMErelated signature between the high- and low-risk groups, gene set enrichment analysis (GSEA) was performed using GSEA software (version: 4.0.3) [16]. Annotated gene sets c2.cp.kegg.v7.2.symbols.gmt and c5.go.v7.2.symbols.gmt were chosen as the reference gene sets. P-value $<0.05$ were considered statistically significant.

The correlation between the prognostic model and tumor-infiltrating immune cells

Different types of tumor-infiltrating immune cells were obtained from Tumor Immune Estimation Resource (TIMER) (https://cistrome.shinyapps.io/timer/) database using gene expression profiles based on different algorithm [17]. We assessed the correlation of the prognostic model between with stromal scores, immune scores and tumorinfiltrating immune cells by spearman correlation analysis in $\mathrm{R}$. Then, we compared the fraction of 22 immune cell types between low- and high-risk groups using the Wilcoxon rank sum test. $\mathrm{P}$ value $<0.05$ were considered statistically significant.

\section{Results}

\section{Relationship of immune/stromal scores with clinicopathological indicators and the prognosis in patients with CCA}

The overall scheme of this study is shown in the Figure 1. A total of 32 CCA patients were collected from TCGA database according to the screening criteria. Immune/stromal scores of the $32 \mathrm{CCA}$ patients were calculated based on the ESTIMATE algorithm using gene expression profile. We firstly analyzed the distribution of immune/stromal scores in different group with clinical characteristics. Our results showed that there was no significant correlation between immune/stromal scores and clinicopathological indicators, such as tumor grade and stage (Data not shown). Next, we divided the 32 CCA patients into high- and low-score groups according to the median immune/stromal scores to detect the potential correlation between survival and immune/stromal scores (Figures 2A-D). Kaplan-Meier survival 
analysis showed that low immune scores $(\mathrm{p}=0.0320)$ (Figure $2 \mathrm{C}$ ) were associated with poorer overall survival (OS) than those with high scores.

\section{Identification of DEGs in CCA based on immune/stromal scores}

To identify the DEGs, differential expression analysis was performed between highand low-score groups using the Dseq2 package in R. With the threshold of $|\operatorname{logFC}|>1$ and FDR $<0.05,745$ upregulated and 142 downregulated immune-related genes were obtained based on immune scores (Figure 3A, Table S1). In addition, we identified 791 upregulated and 171 downregulated stromal-related genes based on stromal scores (Figure 3B, Table S2). Hierarchical clustering analysis results visualized the difference in expression patterns of the top 20 up-regulated and down-regulated DEGs between high- and low-stromal/immune-score groups (Figures 3C, D). Furthermore, Venn diagrams showed that 674 genes were commonly upregulated and 110 genes were commonly downregulated in the high-score groups (Figures 3E, F).

\section{Functional enrichment analysis and PPI network}

To investigate the biological characteristics of DEGs, functional enrichment analyses were performed, including GO enrichment and KEGG pathway analysis. The GO results showed that DEGs were mainly involved in "T cell activation", "lymphocyte proliferation and differentiation", "MHC protein complex", "cytokine binding" and "chemokine binding" (Figure 4A). Furthermore, KEGG enrichment analysis results indicated that these DEGs were mainly involved in "cell adhesion molecular", "chemokine signaling", "hematopoietic cell lineage" and "Th17 cell differentiation" (Figure 4B). All the results of GO and KEGG pathway analysis were showed in Table S3 and Table S4. To explore the relationships between these DEGs, we performed PPI network analysis using STRING database and Cytoscape software. Top 3 most significant modules were selected from the PPI network using the MCODE plugin in Cytoscape software (Figures 4C-E). these genes in the top 3 significant modules were selected for further analysis. These results of GO and KEGG pathway analysis indicated that these genes in the top 3 significant modules were strongly associated with the immune response (Figures S1A-B).

\section{Identification of TME-related prognostic genes and construction of a two-gene risk prediction model}

In order to evaluate the prognostic effect of DEGs, univariate Cox regression analysis was performed, and 22 survival-related DEGs were identified with $\mathrm{P}<0.05$ (Table 2). Subsequently, we conducted a LASSO regression and multivariate Cox regression, two genes were identified and used to construct a novel TME-related prognostic model for CCA (Figures 5A, B). As shown in forest plots, KLRB1 was a protective factor, while GAD1 was risk factor for CCA (Figure 5C). After extracting the multivariate Cox regression coefficient, we calculated risk score of each patient on the basis of the following formula: risk Score $=(0.2940 *$ expression value of GAD1 $)+(-0.5259 *$ expression value of KLRB1). Patients were categorized into two groups according to 
the median risk score, namely the high-risk group and the low-risk group. Kaplan-Meier survival analysis showed that patients with high-risk score had shorter survival time than those with low-risk score (Figure 5D). The AUC value of ROC curve analysis for predicting the survival of patients at 1-, 2-, and 3-years was $0.811,0.772$ and 0.844 , respectively (Figures 5E-G), indicated that the TME-related prognostic model was relatively accurate. Risk score curve and survival status plot reflecting that the number of death cases in high-risk group is significantly more than that in the low-risk group (Figures 5H, I). The heatmap showed the expression of two-gene signature between high- and low-risk groups (Figure 5J).

\section{Independent prognostic analysis}

In order to examine the clinical independence of the model, we performed univariate/multivariate independent prognostic analysis. Univariate independent prognostic analysis (Figure 6A) showed that high-risk score was related to poor prognosis $(\mathrm{HR}=2.718, \mathrm{p}<0.001)$. Moreover, multivariate independent prognostic analysis (Figure 6B) indicated that the risk score could serve as an independent prognostic factors for patients with CCA $(\mathrm{HR}=3.21, \mathrm{p}=0.003)$.

\section{GSEA of the mechanism underlying the prognostic differences between the two groups}

In order to investigated the potential molecular mechanisms of the prognosis difference between high- and low-risk score, we performed GSEA analysis. GO terms enrichment in the high-risk score group was mainly focused on: "Golgi cisterna membrane" and "positive regulation of smoothened signaling pathway" (Figure7A), and KEGG pathway enrichment was in the high-risk score group was mainly involved in notch signaling pathway (Figure 7B). However, in the low-risk score group, GO enrichment was primarily focused on: "regulation of cardiac muscle contraction by regulation of the release of sequestered calcium ion", "negative regulation of NF-kappaB transcription factor activity" and "CD4-positive and alpha-beta $\mathrm{T}$ cell cytokine production" (Figure 7C); and in the case of KEGG pathway, cell adhesion molecules pathway was significantly enriched (Figure 7D). Results of the GSEA are shown in Table S5.

\section{Correlation of risk score to TME-score and immune cell infiltration}

To evaluate the correlation between the TME score (including immune/stromal/ESTIMATE score) and the prognostic model, spearman correlation test was performed. The results showed that the risk score of the prognostic model was negative correlated with the immune/stromal/ESTIMATE score (Figures 8A-C).

We evaluated the correlations between the prognostic model and tumor-infiltrating immune cells which were obtained from TIMER database through different algorithm. Based on TIMER algorithm, we analyzed the relationship between the prognostic model and six type of tumor-infiltrating immune cells. Our results showed that B cells 
$($ Cor $=-0.462, \mathrm{P}=7.83 \mathrm{e}-03)$ (Figure 9A) and CD4+ T cells $($ Cor $=-0.467, \mathrm{P}=7 \mathrm{e}-03$ )

(Figure 9B) were negative correlated with risk score. However, there were no significant correlations between the risk score and dendritic cell (Figure 9C), neutrophil (Figure 9D), CD8+ T cells (Figure 9E) and macrophage (Figure 9F). In addition, we compared the difference of 22 type of tumor-infiltrating immune cells, which obtained from TIMER database through CIBERSORT algorithm between highand low-risk groups. Our results showed that the abundance of naïve B cell $(p=0.04)$ and CD4 memory resting $\mathrm{T}$ cell $(\mathrm{p}=0.0063)$ were significantly enriched in low-risk group as compared to the high-risk group (Figure 10A). Finally, we performed spearman correlation test to analyze the correlations between 20 different immune cell types. The correlation of the 20 different immune cells was range from -0.64 to 0.86 , the correlation coefficient between resting mast cell and resting NK cell was 0.86 , indicating that resting mast cell was significantly correlated with resting NK cell. The correlation of the other immune cells was range -0.64 to 0.57 , which indicates that they were weakly to moderately correlated (Figure 10B).

\section{Survival and expression analysis of the TME-related prognostic signature in CCA patients}

To analyze the survival and expression of the two-gene signature in CCA patients, we integrated mRNA expression profile of the two signature genes and survival information of CCA patients. Our results showed that the expression of GAD1 mRNA was significantly upregulated in tumor tissues ( $\mathrm{P}<0.0001)$ (Figure 11A), while KLRB1 mRNA expression was significantly decreased in tumor tissues $(\mathrm{P}=0.0046)$ (Figure 11C). The Kaplan-Meier survival analysis demonstrated that Patients with high GAD1 expression had poorer overall survival $(\mathrm{P}=8.25 \mathrm{e}-03$; Figure 11B), and those with low KLRB1 expression had poorer overall survival ( $p=1.637 \mathrm{e}-02$; Figure 11D). Therefore, GAD1 may be a promising therapeutic target for CCA patients.

\section{Discussion}

Cholangiocarcinomas are aggressive tumors, and most patients were diagnosed at advanced stage [1]. For patients with advanced-stage or unresectable cholangiocarcinoma, the overall survival of untreated patients is a dismal median of 3.9 months [4]. Although several combination therapeutic regimens for CCA have been applied into clinical practice to improve prognosis, the median overall survival is still less than 1 year [4]. Thus, identification of new biomarkers and establishing prediction models of CCA for diagnosis and treatment are much warranted. Accumulating evidence suggests that the TME plays an important role in occurrence and development of tumors and targeting TME has large potential for anti-cancer treatment. Recently, TME-related signature has gained much attention and shown great potential in prognosis prediction of cancer. Therefore, it is critical to screen prognostic markers related to TME of CCA. 
In this study, we first calculated the stromal and immune scores of CCA tumor tissues using the ESTIMATE algorithm and analyzed the relationship of stromal-immune scores between with prognosis of CCA patients. We found that CCA patients with low immune/stromal scores had a poorer prognosis than those with high scores, which indicates that the TME composition affects the clinical outcomes of CCA patients. Then, a total of 784 overlapping DEGs based on immune and stromal score were obtained for further analysis. Function enrichment analysis of these DEGs and the genes in the top 3 modules obtained from PPI network showed both the GO terms and KEGG pathways were strongly associated with the immune response.

Furthermore, we identified a two-gene signature (including GAD1 and KLRB1) and subsequently established a novel risk prediction model for CCA prognosis prediction. Among them, glutamic acid decarboxylase 1 (GAD1) serves as a rate-limiting enzyme involved in synthesizing $\gamma$-aminobutyric acid (GABA). Over expression of GAD1 has been found in several cancers (including oral cancer, colorectal carcinoma, breast cancer, lymphoma and gastric cancer) and it can influence the proliferation, metastasis and chemoresistance of cancer cells [18-20]. Increasing research suggests that GAD1 has the potential to be a biomarker for prognosis prediction [21-24]. Tsuboi et al. found that upregulation of GAD1 in lung adenocarcinoma patients showed significantly poorer prognosis for overall survival [22]. Lee et al. reported overexpression of GAD1 as a poor prognostic factor in patients with nasopharyngeal carcinoma [23]. Additionally, GAD1 also has been found to be a prognostic marker for glioblastoma (GBM) patients [24]. However, the role and mechanism of GAD1 in CCA remains poorly defined. Killer cell lectin-like receptor B1 (KLRB1), also named as CD161, has been reported as a prognostic gene in many cancers [25-27]. For example, Qin et al. showed that high expression of KLRB1 was associated with good overall survival in early cervical squamous cell carcinoma patients [25]. Braud et al. have shown that expression KLRB1 in lung cancer is associated with better clinical outcome [27]. Consistent with these findings, our studies have demonstrated that low KLRB1 expression in CCA patients had poorer overall survival. However, single-cell analysis in glioma revealed KLRB1 as a candidate inhibitory receptor of tumor-infiltrating T cells, and targeting KLRB1 could enhances T cell-mediated killing of glioma cells [28]. In addition, single-cell analysis in early-relapse hepatocellular carcinoma revealed that CD8+ $\mathrm{T}$ cells in recurrent tumors overexpressed KLRB1 have an innate-like, low cytotoxic, and low clonal expansion phenotype, and these T cell with high KLRB1 expression enrichment in HCC was associated with a worse prognosis [29]. The bimodal role of KLRB 1 in prognosis prediction may be due to the great heterogeneity between different cancers.

Then, we calculated the risk score of each patient based on the TME-related gene signature, and CCA patients were divided into high- and low-risk groups according to the median risk score. Further analysis revealed that the two-gene risk model can be served as an independent prognostic factor in CCA, and patients in the high-risk group showed worse prognosis. ROC curve analysis indicated that the prognostic signature had an excellent performance in predicting OS. Furthermore, we evaluated the 
correlations between the prognostic model and the TME scores. Our results found that the risk score of the prognostic model was negatively correlated with the immune scores, which consistent with the previous results of this research. Both low-risk group and high-immune score group are associated with favorable prognosis, indicating that immune cell infiltration of the TME could have a beneficial impact on prognosis.

Finally, we analysis tumor-infiltrating immune cells of CCA patients using TIMER database based on TIMER and CIBERSORT algorithm, then evaluated the correlations between the prognostic model and tumor-infiltrating immune cells. Our results showed that $\mathrm{B}$ cells $(\mathrm{Cor}=-0.462, \mathrm{P}=7.83 \mathrm{e}-03)$ and $\mathrm{CD} 4+\mathrm{T}$ cells $(\mathrm{Cor}=-0.467, \mathrm{P}=7 \mathrm{e}-03)$ were negative correlated with risk score. Further analysis revealed that naïve $\mathrm{B}$ cell (a subtype of the B cell) and CD4 memory resting T cell (a subtype of CD4+ T cell) were enrichment in low-risk group. Previous study revealed that naïve B cell and CD4 memory resting $\mathrm{T}$ cell infiltration was positively correlated with prognosis in several cancers [30-32]. Thus, infiltrating of naïve B cell and CD4 memory resting T cell may play important roles in the prognosis of CCA patients, which will be worth further investigating.

However, this study also had some limitations. First, the sample size of CCA in TCGA database was relatively small, it should be validated in a large sample size and other databases. Second, the function and mechanism of the gene signature in our study was analyzed based on multiple bioinformatics tools, the conclusion of this study need to be further confirmed by a series of basic experiments.

\section{Conclusions}

In conclusion, our study established a novel TME-related gene signature to predict the prognosis of patients with CCA. This may further our understanding of the potential relationship between TME and CCA prognosis, and serve as a prognosis stratification tool for guiding personalized treatment of CCA patients.

\section{Abbreviations}

CCA: Cholangiocarcinoma; TME: Tumor microenvironment; GSEA: gene set enrichment analyses; TIMER: Tumor Immune Estimation Resource; DEGs: Differently expressed genes; ESTIMATE: Estimation of STromal and Immune cells in Malignant Tumors using Expression data; TCGA: The Cancer Genome Atlas; PPI: protein-protein interaction; GO: Gene Ontology; KEGG: Kyoto Encyclopedia of Genes and Genomes; STRING: Search Tool for the Retrieval of interacting Genes; MCODE: Molecular Complex Detection; GSEA: Gene set enrichment analysis; OS: Overall survival.

\section{Acknowledgements}

Not applicable.

\section{Authors' contributions}


YB and HY designed the study and revised the manuscript. JC, $\mathrm{XC}$ and $\mathrm{XZ}$ collected the data. JC analysed the data. JC and HW drafted the manuscript. GL revised the manuscript. All authors read and approved the final manuscript.

\section{Funding}

This work was supported by the National Natural Science Foundation of PR China Grants 81874166, 32071413, 31471274 and 81502393.

\section{Availability of data and materials}

Gene expression profile and clinical data of CCA patients were downloaded from The Cancer Genome Atlas (TCGA) database (https://portal.gdc.cancer.gov/).

\section{Declarations}

Ethics approval and consent to participate

Not applicable.

\section{Consent for publication}

Not applicable.

\section{Competing interests}

All authors declare no conflict of interests.

\section{References}

1. Krasinskas AM: Cholangiocarcinoma. Surg Pathol Clin 2018, 11(2):403-429.

2. Rizvi S, Khan SA, Hallemeier CL, Kelley RK, Gores GJ: Cholangiocarcinoma - evolving concepts and therapeutic strategies. Nat Rev Clin Oncol 2018, 15(2):95-111.

3. Morimoto Y, Tanaka Y, Ito T, Nakahara M, Nakaba H, Nishida T, Fujikawa M, Ito $\mathrm{T}$, Yamamoto $\mathrm{S}$, Kitagawa $\mathrm{T}$ : Long-term survival and prognostic factors in the surgical treatment for intrahepatic cholangiocarcinoma. $J$ Hepatobiliary Pancreat Surg 2003, 10(6):432-440.

4. Choi SB, Kim KS, Choi JY, Park SW, Choi JS, Lee WJ, Chung JB: The prognosis and survival outcome of intrahepatic cholangiocarcinoma following surgical resection: association of lymph node metastasis and lymph node dissection with survival. Ann Surg Oncol 2009, 16(11):30483056.

5. Pitt JM, Marabelle A, Eggermont A, Soria JC, Kroemer G, Zitvogel L: Targeting the tumor microenvironment: removing obstruction to anticancer immune responses and immunotherapy. Ann Oncol 2016, 27(8):1482-1492. 
6. Zhang T, Nie Y, Xia H, Zhang Y, Cai K, Chen X, Li H, Wang J: Identification of Immune-Related Prognostic Genes and LncRNAs Biomarkers Associated With Osteosarcoma Microenvironment. Front Oncol 2020, 10:1109.

7. Yue $\mathrm{C}$, Ma $\mathrm{H}$, Zhou Y: Identification of prognostic gene signature associated with microenvironment of lung adenocarcinoma. PeerJ 2019, 7:e8128.

8. Zhang D, Qian C, Wei H, Qian X: Identification of the Prognostic Value of Tumor Microenvironment-Related Genes in Esophageal Squamous Cell Carcinoma. Front Mol Biosci 2020, 7:599475.

9. Liu X, Niu X, Qiu Z: A Five-Gene Signature Based on Stromal/Immune Scores in the Tumor Microenvironment and Its Clinical Implications for Liver Cancer. DNA Cell Biol 2020, 39(9):1621-1638.

10. Qi X, Qi C, Qin B, Kang X, Hu Y, Han W: Immune-Stromal Score Signature: Novel Prognostic Tool of the Tumor Microenvironment in Lung Adenocarcinoma. Front Oncol 2020, 10:541330.

11. Wang H, Wu X, Chen Y: Stromal-Immune Score-Based Gene Signature: A Prognosis Stratification Tool in Gastric Cancer. Front Oncol 2019, 9:1212.

12. Vigano L, Soldani C, Franceschini B, Cimino M, Lleo A, Donadon M, Roncalli M, Aghemo A, Di Tommaso L, Torzilli G: Tumor-Infiltrating Lymphocytes and Macrophages in Intrahepatic Cholangiocellular Carcinoma. Impact on Prognosis after Complete Surgery. J Gastrointest Surg 2019, 23(11):22162224.

13. Yoshihara K, Shahmoradgoli M, Martinez E, Vegesna R, Kim H, Torres-Garcia W, Trevino V, Shen H, Laird PW, Levine DA et al: Inferring tumour purity and stromal and immune cell admixture from expression data. Nat Commun 2013, 4:2612.

14. Szklarczyk D, Gable AL, Lyon D, Junge A, Wyder S, Huerta-Cepas J, Simonovic M, Doncheva NT, Morris JH, Bork P et al: STRING v11: proteinprotein association networks with increased coverage, supporting functional discovery in genome-wide experimental datasets. Nucleic Acids Res 2019, 47(D1):D607-D613.

15. Shannon P, Markiel A, Ozier O, Baliga NS, Wang JT, Ramage D, Amin N, Schwikowski B, Ideker T: Cytoscape: a software environment for integrated models of biomolecular interaction networks. Genome Res 2003, 13(11):2498-2504.

16. Subramanian A, Tamayo P, Mootha VK, Mukherjee S, Ebert BL, Gillette MA, Paulovich A, Pomeroy SL, Golub TR, Lander ES et al: Gene set enrichment analysis: a knowledge-based approach for interpreting genome-wide expression profiles. Proc Natl Acad Sci U S A 2005, 102(43):15545-15550.

17. Li T, Fu J, Zeng Z, Cohen D, Li J, Chen Q, Li B, Liu XS: TIMER2.0 for analysis of tumor-infiltrating immune cells. Nucleic Acids Res 2020, 48(W1):W509-W514.

18. Schnepp PM, Lee DD, Guldner IH, O'Tighearnaigh TK, Howe EN, Palakurthi B, Eckert KE, Toni TA, Ashfeld BL, Zhang S: GAD1 Upregulation Programs 
Aggressive Features of Cancer Cell Metabolism in the Brain Metastatic Microenvironment. Cancer Res 2017, 77(11):2844-2856.

19. Yan H, Tang G, Wang H, Hao L, He T, Sun X, Ting AH, Deng A, Sun S: DNA methylation reactivates GAD1 expression in cancer by preventing CTCFmediated polycomb repressive complex 2 recruitment. Oncogene 2016, 35(30):3995-4008.

20. Kimura R, Kasamatsu A, Koyama T, Fukumoto C, Kouzu Y, Higo M, EndoSakamoto Y, Ogawara K, Shiiba M, Tanzawa $\mathrm{H}$ et al: Glutamate acid decarboxylase 1 promotes metastasis of human oral cancer by beta-catenin translocation and MMP7 activation. BMC Cancer 2013, 13:555.

21. Jaraj SJ, Augsten M, Haggarth L, Wester K, Ponten F, Ostman A, Egevad L: GAD1 is a biomarker for benign and malignant prostatic tissue. Scand $J$ Urol Nephrol 2011, 45(1):39-45.

22. Tsuboi M, Kondo K, Masuda K, Tange S, Kajiura K, Kohmoto T, Takizawa H, Imoto I, Tangoku A: Prognostic significance of GAD1 overexpression in patients with resected lung adenocarcinoma. Cancer Med 2019, 8(9):41894199.

23. Lee YY, Chao TB, Sheu MJ, Tian YF, Chen TJ, Lee SW, He HL, Chang IW, Hsing $\mathrm{CH}$, Lin $\mathrm{CY}$ et al: Glutamate Decarboxylase 1 Overexpression as a Poor Prognostic Factor in Patients with Nasopharyngeal Carcinoma. $J$ Cancer 2016, 7(12):1716-1723.

24. Wong KK, Rostomily R, Wong STC: Prognostic Gene Discovery in Glioblastoma Patients using Deep Learning. Cancers (Basel) 2019, 11(1).

25. Qin R, Cao L, Ye C, Wang J, Sun Z: A novel prognostic prediction model based on seven immune-related RNAs for predicting overall survival of patients in early cervical squamous cell carcinoma. BMC Med Genomics 2021, 14(1):49.

26. Ye $\mathrm{Q}, \mathrm{Han} \mathrm{X}, \mathrm{Wu} \mathrm{Z}$ : Bioinformatics analysis to screen key prognostic genes in the breast cancer tumor microenvironment. Bioengineered 2020, 11(1):1280-1300.

27. Braud VM, Biton J, Becht E, Knockaert S, Mansuet-Lupo A, Cosson E, Damotte D, Alifano M, Validire P, Anjuere F et al: Expression of LLT1 and its receptor CD161 in lung cancer is associated with better clinical outcome. Oncoimmunology 2018, 7(5):e1423184.

28. Mathewson ND, Ashenberg O, Tirosh I, Gritsch S, Perez EM, Marx S, JerbyArnon L, Chanoch-Myers R, Hara T, Richman AR et al: Inhibitory CD161 receptor identified in glioma-infiltrating $T$ cells by single-cell analysis. Cell 2021, 184(5):1281-1298 e1226.

29. Sun Y, Wu L, Zhong Y, Zhou K, Hou Y, Wang Z, Zhang Z, Xie J, Wang C, Chen $\mathrm{D}$ et al: Single-cell landscape of the ecosystem in early-relapse hepatocellular carcinoma. Cell 2021, 184(2):404-421 e416.

30. Zhang Z, Ni Y, Chen G, Wei Y, Peng M, Zhang S: Construction of immunerelated risk signature for uveal melanoma. Artif Cells Nanomed Biotechnol 2020, 48(1):912-919. 
31. Zhao M, Li M, Chen Z, Bian Y, Zheng Y, Hu Z, Liang J, Huang Y, Yin J, Zhan $\mathrm{C}$ et al: Identification of immune-related gene signature predicting survival in the tumor microenvironment of lung adenocarcinoma. Immunogenetics 2020, 72(9-10):455-465.

32. Zhang L, Li B, Peng Y, Wu F, Li Q, Lin Z, Xie S, Xiao L, Lin X, Ou Z et al: The prognostic value of TMB and the relationship between TMB and immune infiltration in head and neck squamous cell carcinoma: A gene expression-based study. Oral Oncology 2020, 110.

\section{Figure legends}

Figure 1. Flow diagram of the study.

Figure 2. Association between immune/stromal score and prognosis of CCA patients in TCGA database. The distribution of immune/stromal score in high and low group (A, B). Kaplan-Meier survival analysis of the OS in high and low groups based on immune score (C) and stromal score (D).

Figure 3. Identification of stromal- and immune-related DEGs. Volcano plots showing the distribution of stromal-related (A) and immune-related (B) DEGs in CCA. DEGs with $|\log \mathrm{FC}|>1$ and FDR $<0.05$. The heatmap depicting the expression patterns of the top 20 up-regulated and down-regulated genes between high and low stromal (C) and immune (D) score groups. Venn diagrams showing the overlapping upregulated (E) and downregulated $(\mathbf{F})$ genes between stromal-related genes and immune-related genes.

Figure 4. Functions of stromal- and immune-related DEGs. (A) GO enrichment analysis, including BP, CC and MF. (B) KEGG pathway enrichment analysis. (C-E) Top 3 modules from a PPI network of 784 significantly differentially expressed immune-related genes.

Figure 5. Identification of Immune-related prognostic genes and construction of a twogene prognostic model. (A, B) LASSO regression analysis. (C) Forest plots of the 4gene signature. (D) Kaplan-Meier survival analysis of the risk score. (E-G) ROC curves of the risk model for predicting 1-, 2- and 3-year survival probability. (H) Dot plot of the risk score. (I) Dot plot of survival. (J) The heatmap showing the expression of the two-gene signature between high-risk and low-risk group.

Figure 6. Univariate and multivariate independent prognostic analysis. Forest plots of univariate (A) and multivariate (B) independent prognostic analysis.

Figure 7. GSEA analysis of the differences between high-risk and low-risk score groups. (A, B) List top 1 of GO terms in the high- and low-risk score groups. (C, D) List top 1 of KEGG pathway enrichment in the high- and low-risk score groups.

Figure 8. The correlation between the risk scores and tumor microenvironment scores. (A) Stromal score; (B) Immune score; (C) ESTIMATE score. 
Figure 9. The correlation between the risk score and infiltration levels of six types of immune cells (A-F). (A) B cells, (B) CD4+ T cells, (C) Dendritic cell, (D) Neutrophil, (E) $\mathrm{CD} 8+\mathrm{T}$ cells, (F) macrophage.

Figure 10. Difference and correlation analysis of tumor-infiltrating immune cells. (A) The difference of tumor-infiltrating immune cells between high- and low-risk score groups. (B) The correlation heatmap of 20 immune infiltration cells in CCA tumor samples.

Figure 11. Expression and survival analysis of the two-gene signature in CCA patients. The mRNA expression levels of GAD1 (A) and KLRB1 (C) in TCGA CCA tumor tissue and normal tissue. (B, D) Kaplan-Meier survival analysis of the correlations between the expression levels of the two-gene signature and the OS.

Supplementary Figure 1. Functional enrichment analysis of these genes in the top 3 modules. (A) Top 10 enriched GO terms of these genes in the top 3 modules. (B) Top 10 enriched KEGG pathways of these genes in the top 3 modules.

Table 1. Clinical features of patients with CCA in TCGA.

Table 2. Univariate Cox regression analysis identified 22 OS-related DEGs in CCA.

Additional file 1. Relationship between immune and stromal scores and CCA clinical characteristics. (A, B) Distributions of immune scores among different ISUP grades. $(C, D)$ Distributions of immune scores among different AJCC stages. 


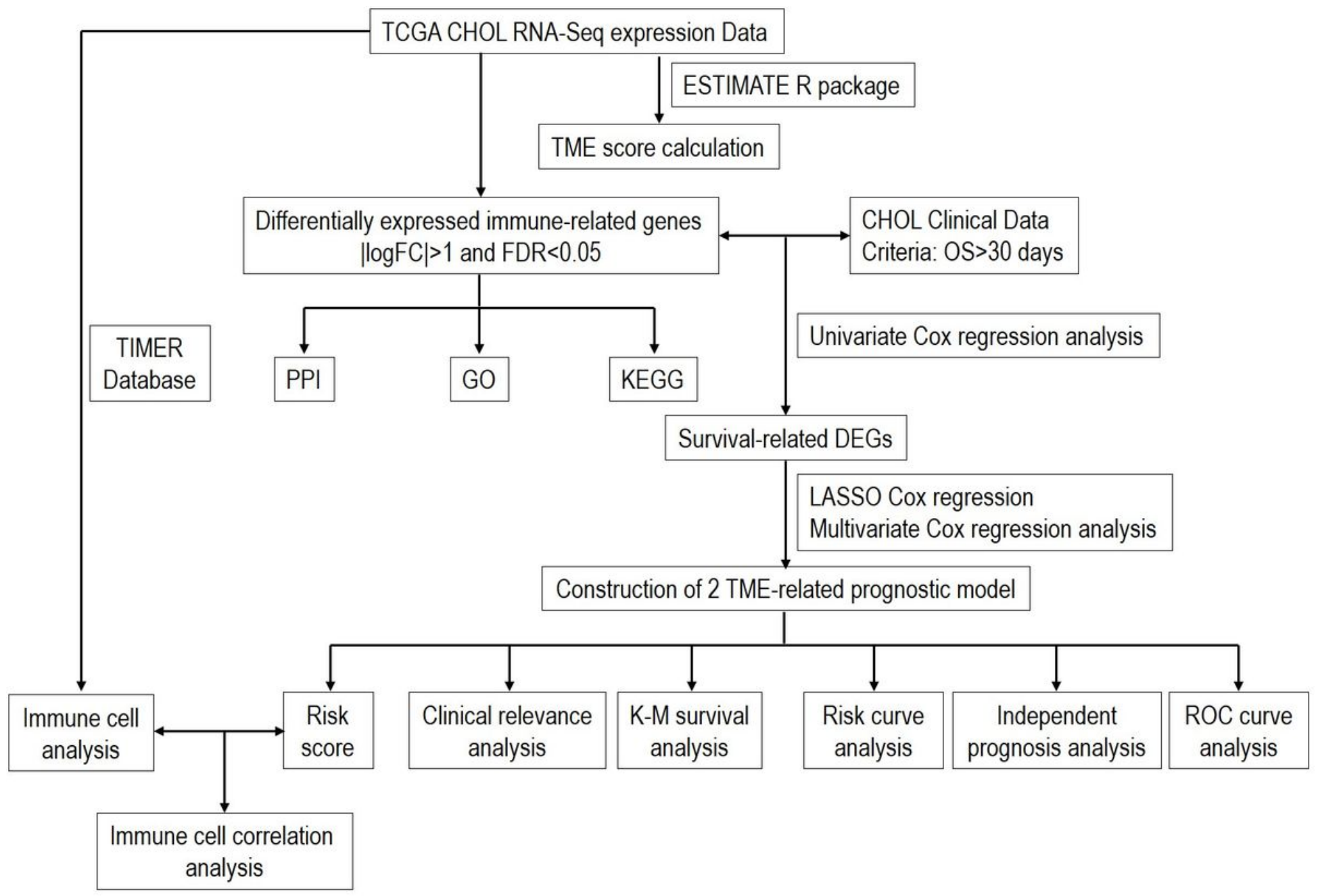

Figure 1

Flow diagram of the study. 
Figure 2 A

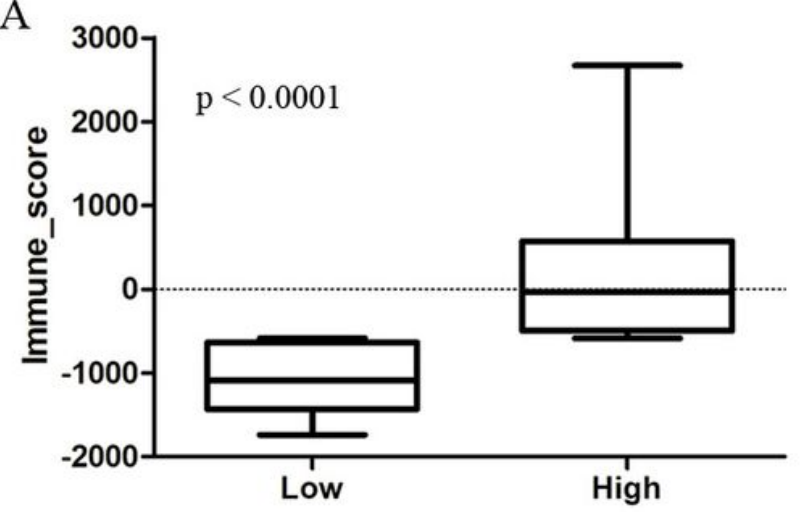

$\mathrm{C}$

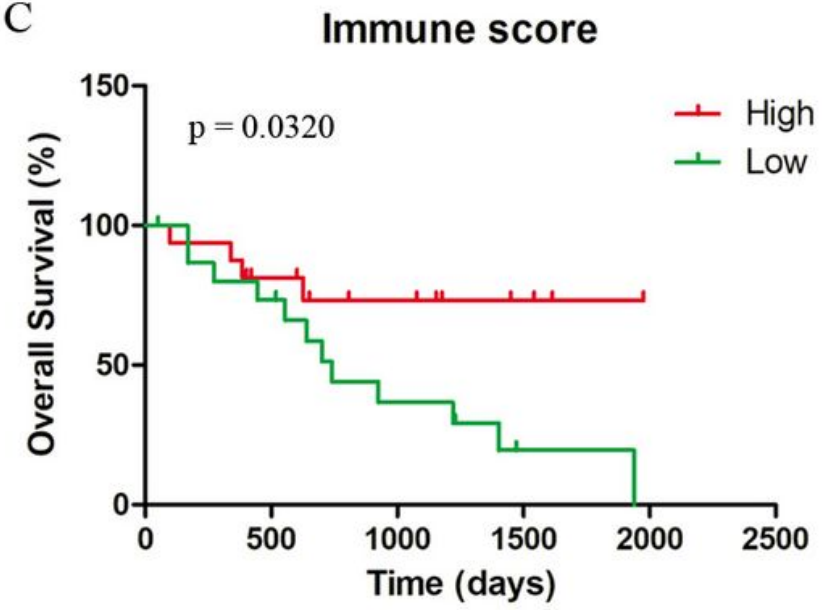

$\mathrm{B}$

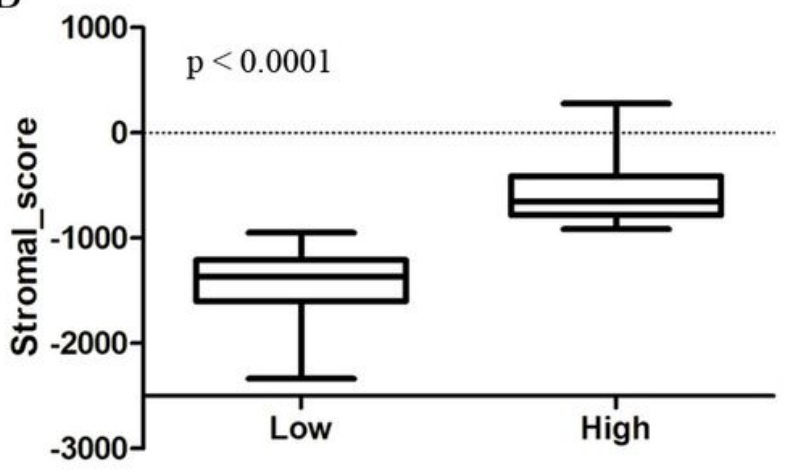

$\mathrm{D}$

Stromal score

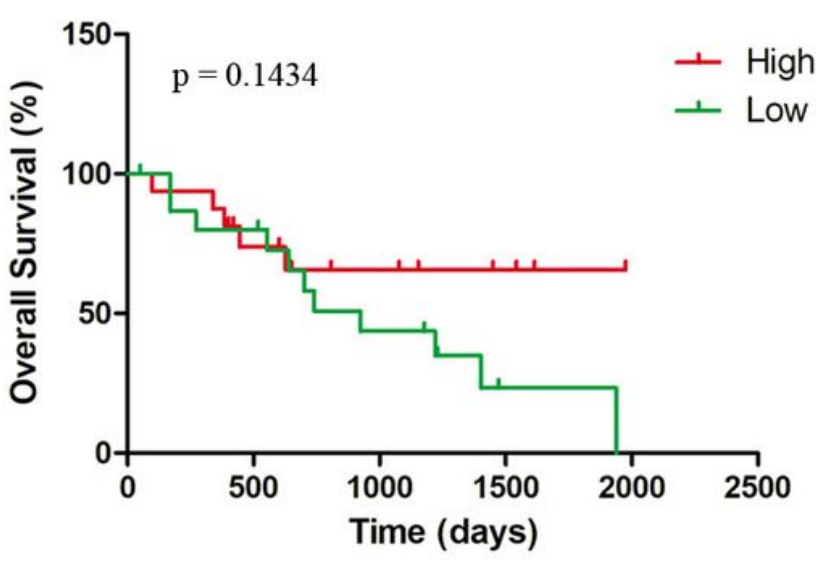

Figure 2

Association between immune/stromal score and prognosis of CCA patients in TCGA database. The distribution of immune/stromal score in high and low group (A, B). Kaplan-Meier survival analysis of the OS in high and low groups based on immune score (C) and stromal score (D). 
Figure 3 A

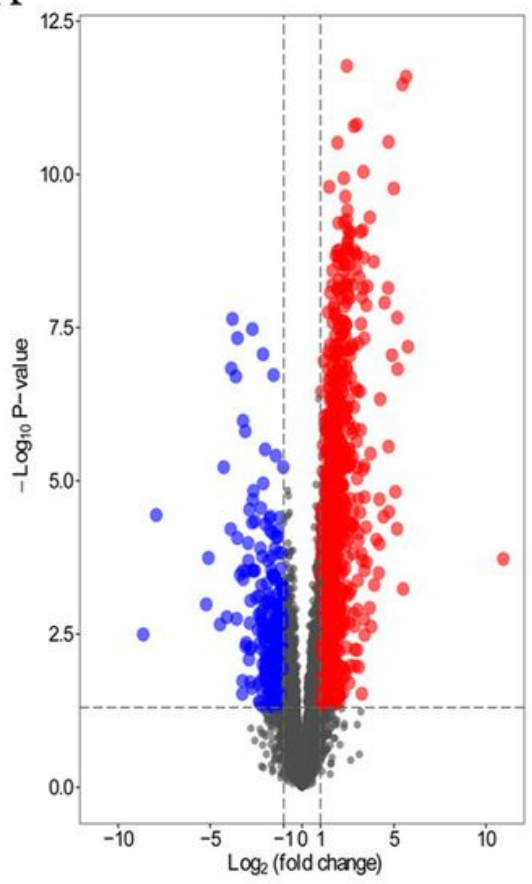

$\mathrm{D}$

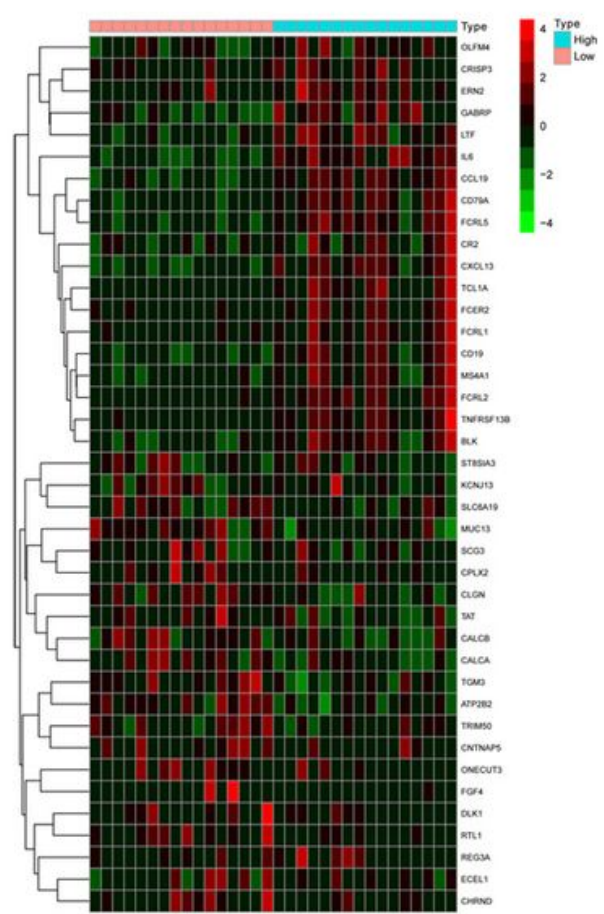

B

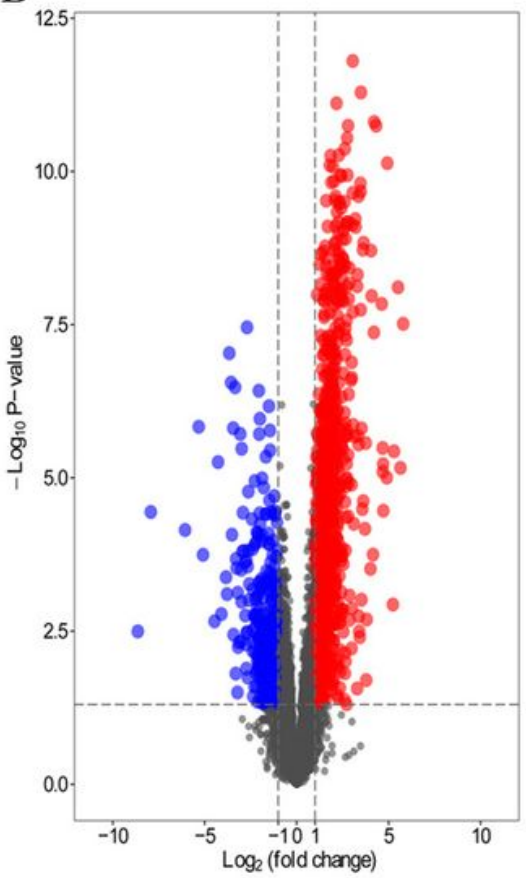

$\mathrm{C}$

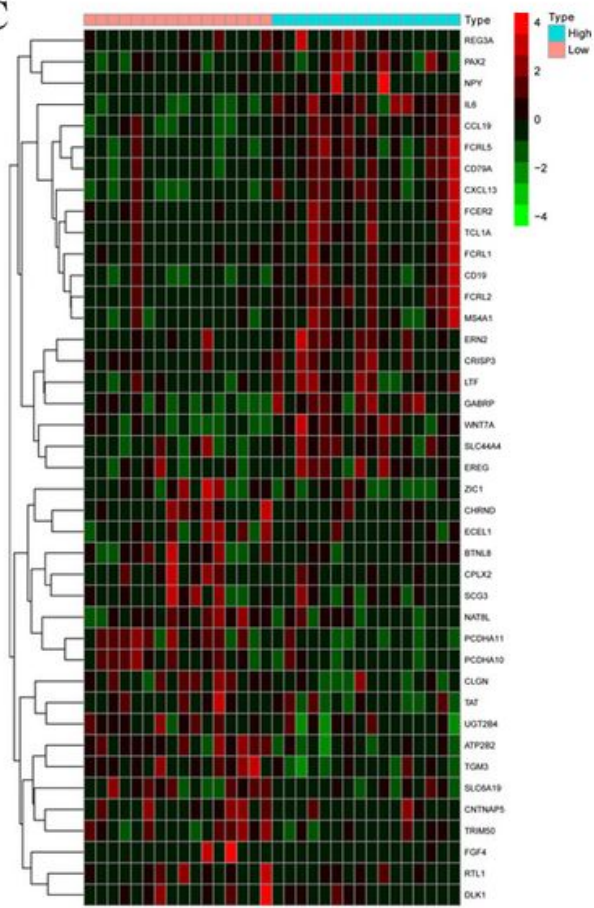

$\mathrm{E}$

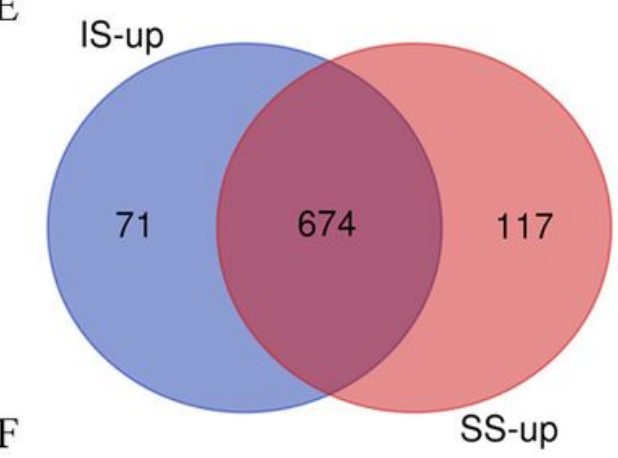

IS-down

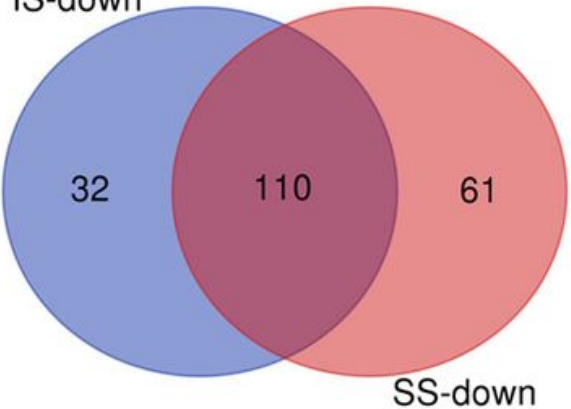

\section{Figure 3}

Identification of stromal- and immune-related DEGs. Volcano plots showing the distribution of stromalrelated (A) and immune-related (B) DEGs in CCA. DEGs with $|\operatorname{logFC}|>1$ and FDR $<0.05$. The heatmap depicting the expression patterns of the top 20 up-regulated and down-regulated genes between high and low stromal (C) and immune (D) score groups. Venn diagrams showing the overlapping upregulated (E) and downregulated $(F)$ genes between stromal-related genes and immune-related genes. 
Figure $4 \mathrm{~A}$

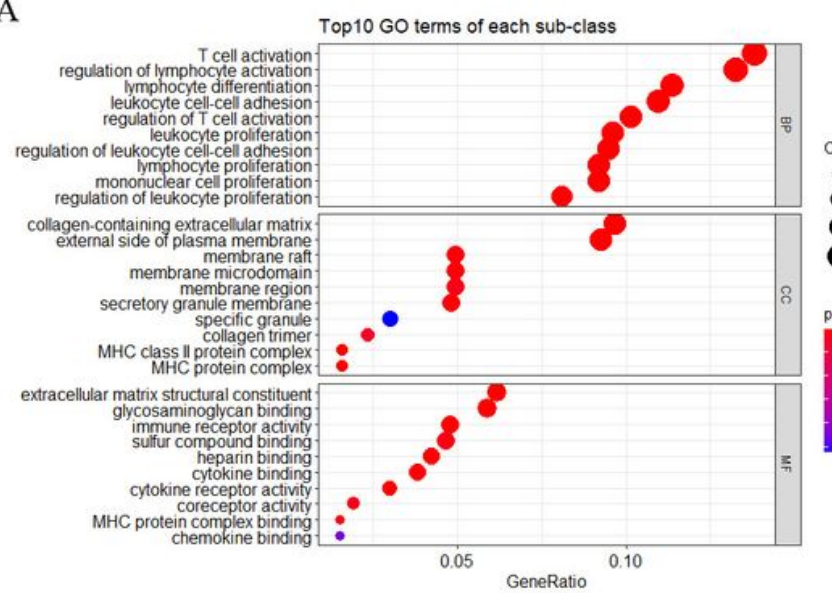

B

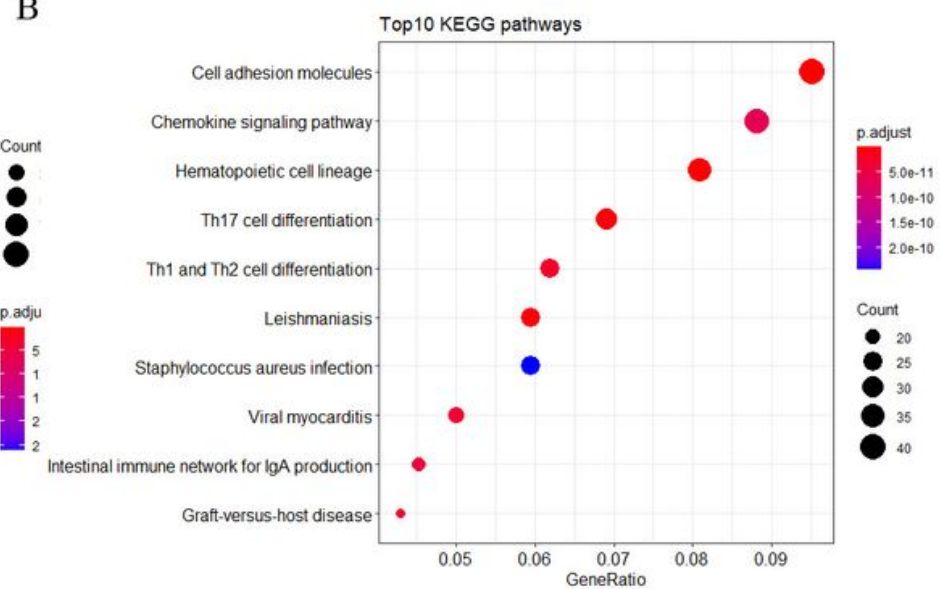

$\mathrm{C}$

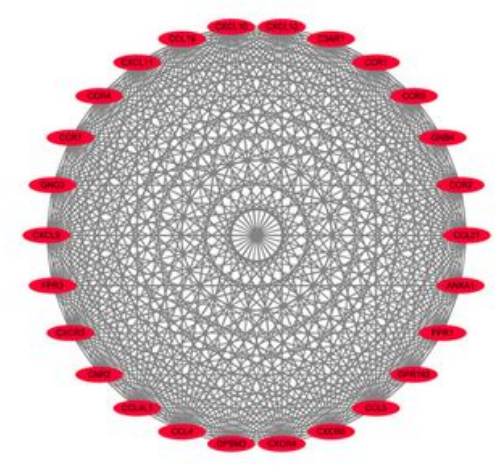

$\mathrm{D}$

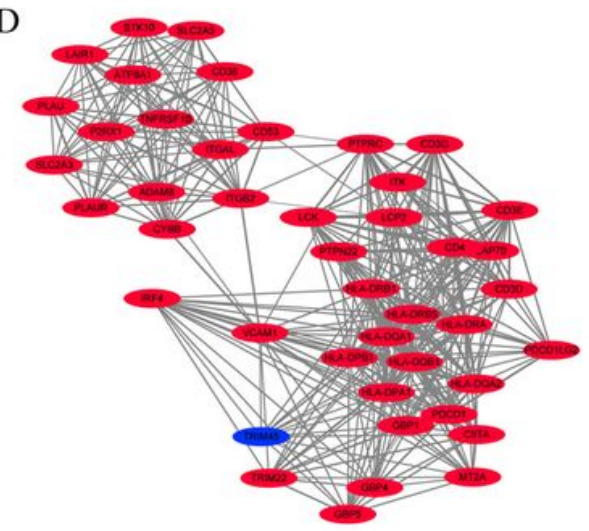

E

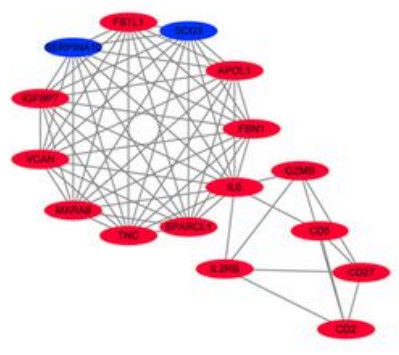

\section{Figure 4}

Functions of stromal- and immune-related DEGs. (A) GO enrichment analysis, including BP, CC and MF. (B) KEGG pathway enrichment analysis. (C-E) Top 3 modules from a PPI network of 784 significantly differentially expressed immune-related genes. 
Figure $5 \mathrm{~A}$

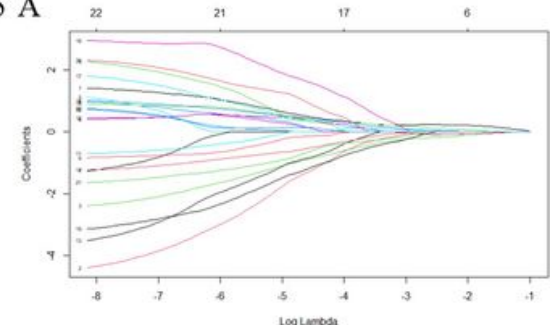

D
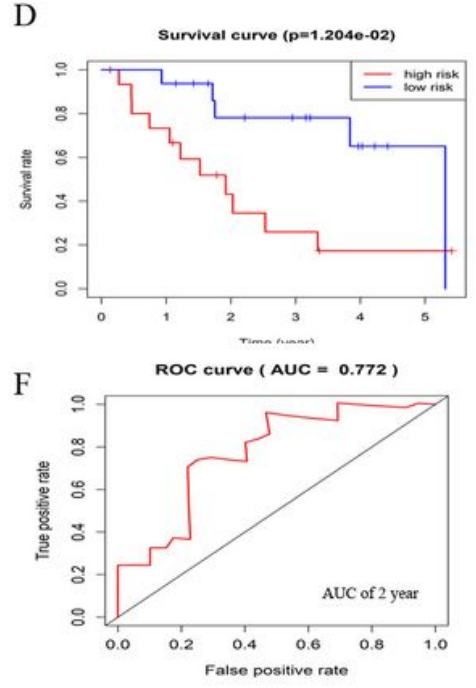

B

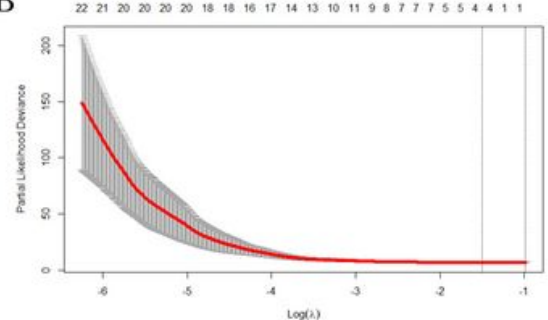

E
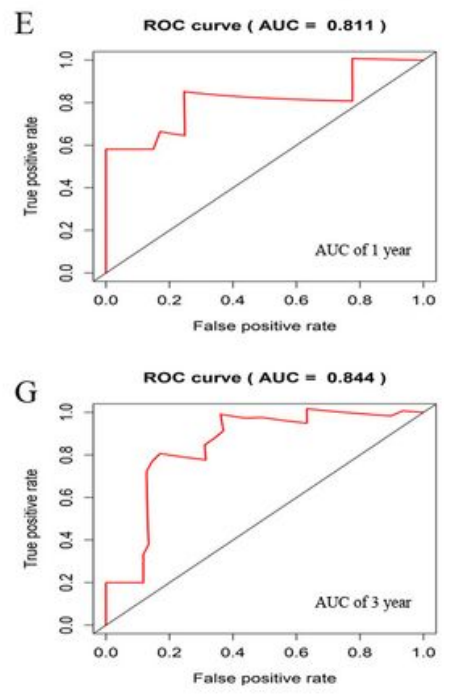

$\mathrm{H}$

I

$\mathrm{J}$
C
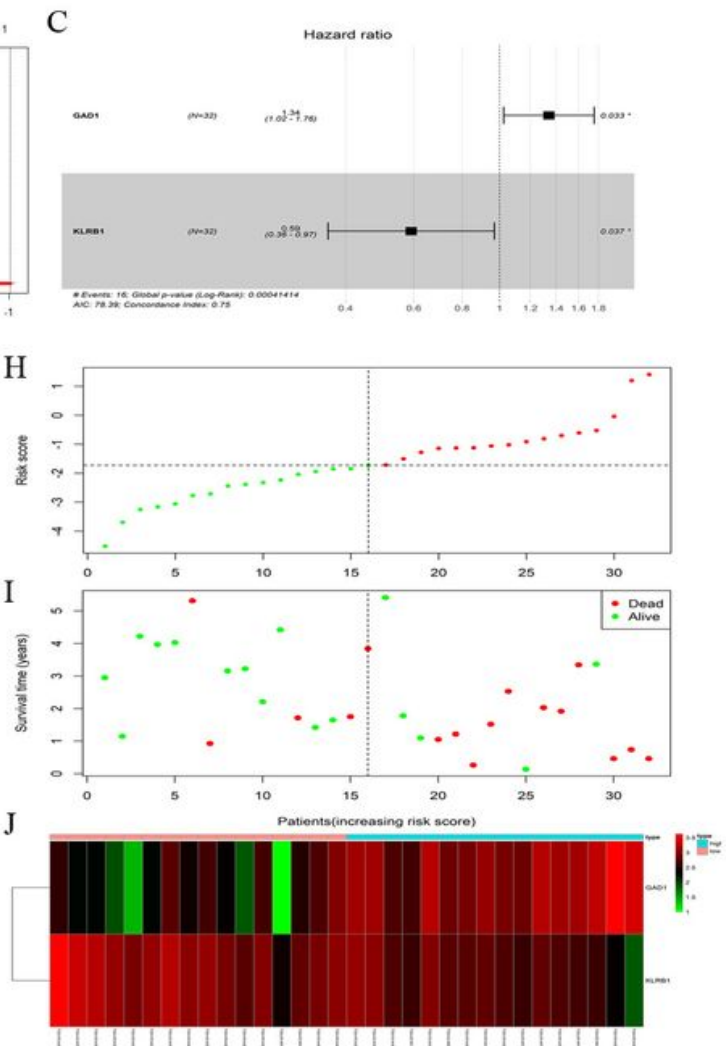

\section{Figure 5}

Identification of Immune-related prognostic genes and construction of a two-gene prognostic model. (A, B) LASSO regression analysis. (C) Forest plots of the 4-gene signature. (D) Kaplan-Meier survival analysis of the risk score. (E-G) ROC curves of the risk model for predicting 1-, 2- and 3-year survival probability. (H) Dot plot of the risk score. (I) Dot plot of survival. (J) The heatmap showing the expression of the two-gene signature between high-risk and low-risk group. 
Figure 6 A

$\begin{array}{lrc}\text { A } & \text { pvalue } & \text { Hazard ratio } \\ \text { age } & 0.511 & 1.013(0.974-1.01) \\ \text { gender(ref. Male) } & 0.568 & 0.749(0.277-2.02) \\ \text { stage(ref. I-II) } & 0.706 & 1.248(0.395-3.94) \\ \text { grade(ref. 1-2) } & 0.713 & 0.82(0.285-2.36) \\ \mathrm{T} \text { (ref. T1) } & 0.415 & 1.528(0.551-4.24) \\ \mathrm{M} \text { (ref. M0) } & 0.739 & 1.241(0.349-4.41) \\ \mathrm{N} \text { (ref. N0) } & 0.03 & 3.266(1.12-9.53) \\ \text { riskScore } & <0.001 & 2.718(1.59-4.65) \\ & \text { pvalue } & \text { Hazard ratio } \\ \text { B } & 0.733 & 1.01(0.957-1.06) \\ \text { age } & 0.789 & 0.85(0.26-2.78) \\ \text { gender(ref. Male) } & 0.902 & 1.19(0.079-17.9) \\ \text { stage(ref. I-II) } & 0.386 & 0.515(0.115-2.31) \\ \text { grade(ref. 1-2) } & 0.686 & 0.734(0.164-3.29) \\ \mathrm{T} \text { (ref. T1) } & 0.555 & 0.516(0.057-4.65) \\ \mathrm{M} \text { (ref. M0) } & 0.84 & 1.23(0.171-8.8) \\ \mathrm{N} \text { (ref. N0) } & 0.003 & 3.21(1.49-6.95) \\ \text { riskScore } & \end{array}$

pvalue Hazard ratio

B

pvalue Hazard ratio

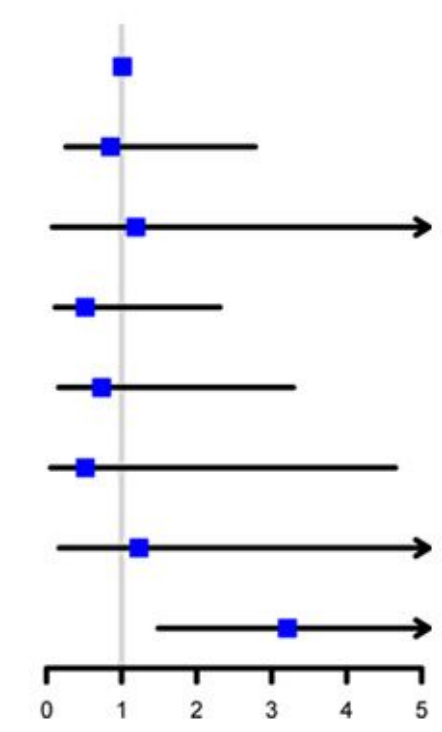

Figure 6

Univariate and multivariate independent prognostic analysis. Forest plots of univariate $(A)$ and multivariate (B) independent prognostic analysis. 
Figure 7 A

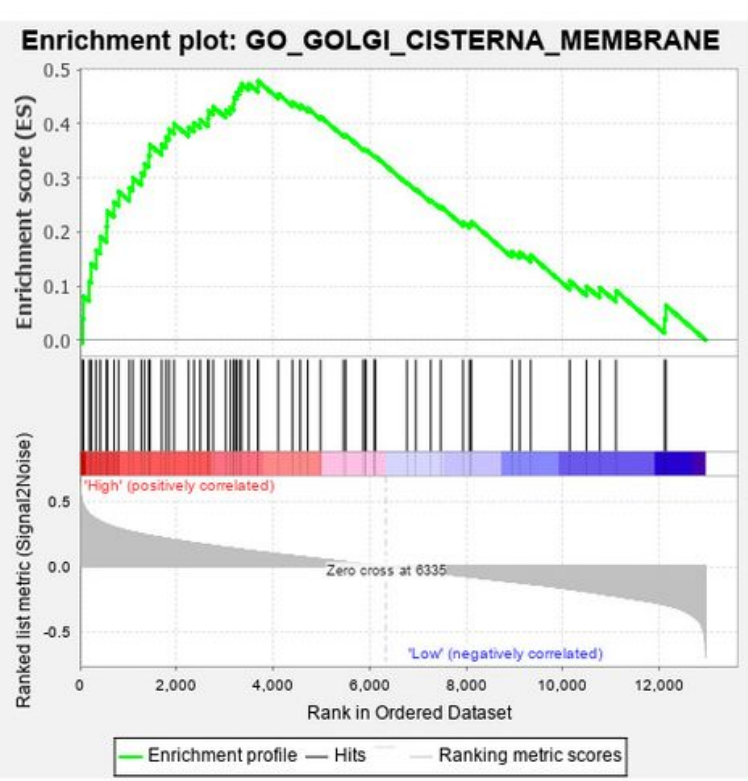

$\mathrm{C}$

Enrichment plot: KEGG_NOTCH_SIGNALING_PATHWAY

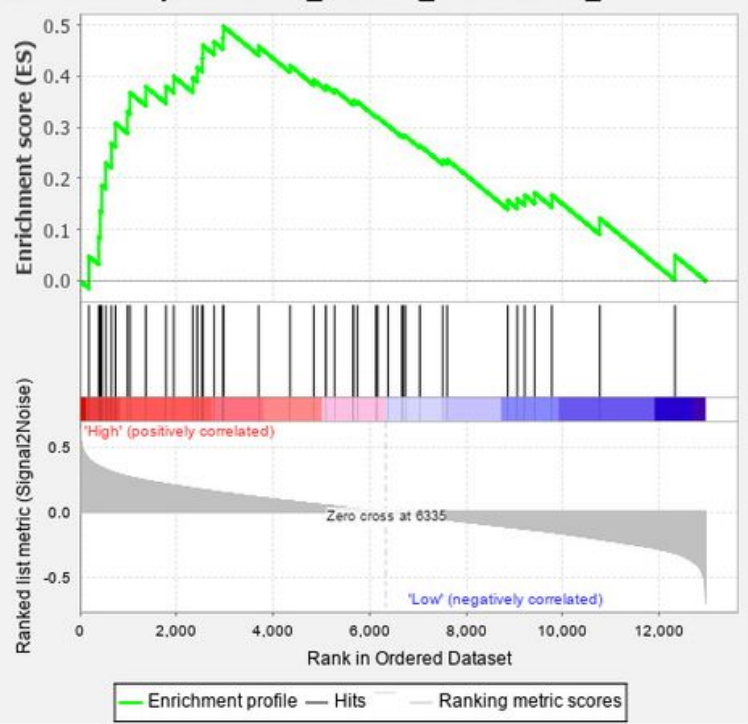

$\mathrm{B}$

Enrichment plot:

GO_REGULATION_OF_CARDIAC_MUSCLE_CONTRACTI ON_BY_REGULATION_OF_THE_RELEASE_OF_SEQUEST ERED_CALCIUM_ION

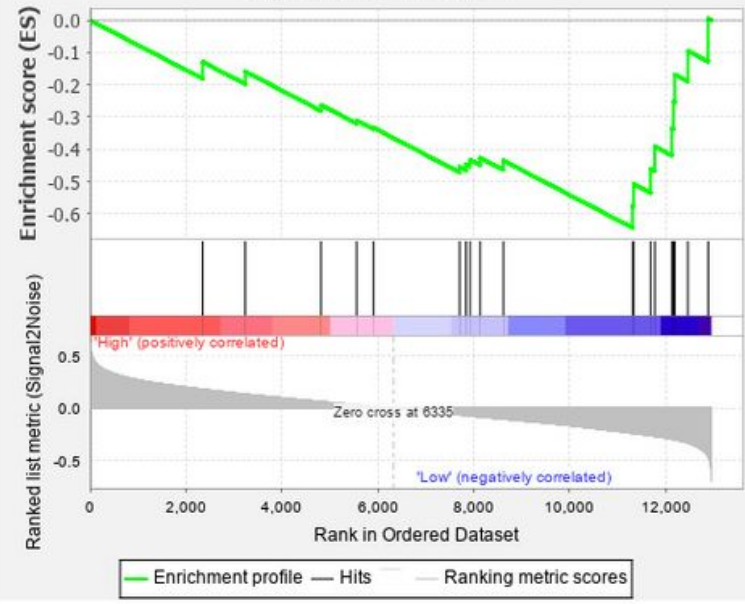

$\mathrm{D}$

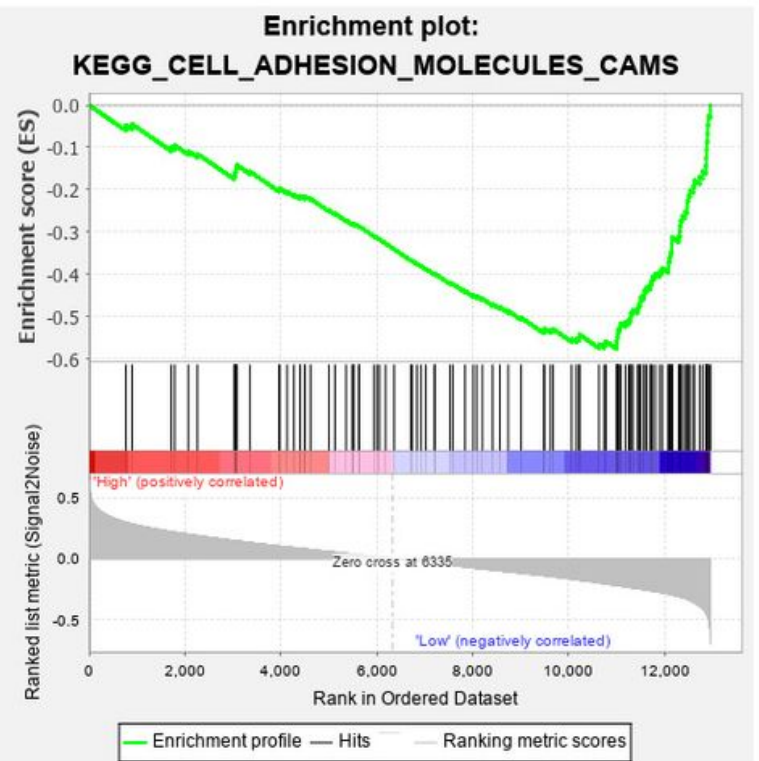

Figure 7

GSEA analysis of the differences between high-risk and low-risk score groups. (A, B) List top 1 of GO terms in the high- and low-risk score groups. (C, D) List top 1 of KEGG pathway enrichment in the highand low-risk score groups. 
Figure $8 \mathrm{~A}$

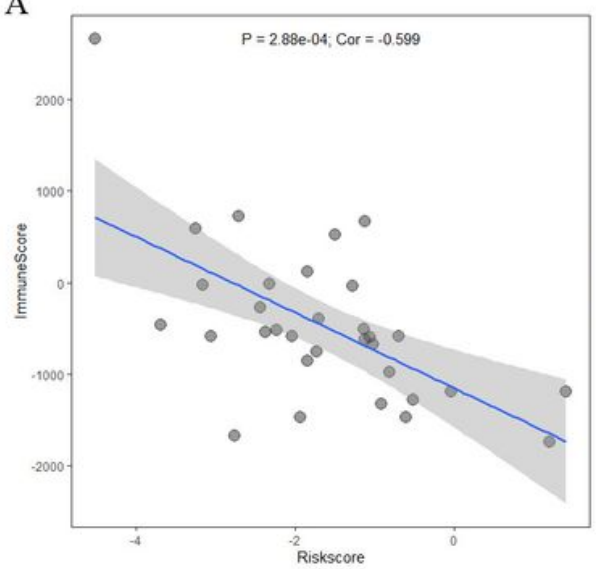

B

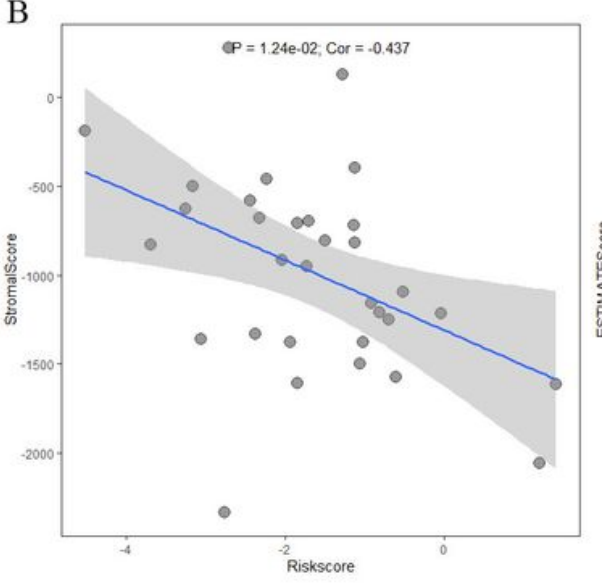

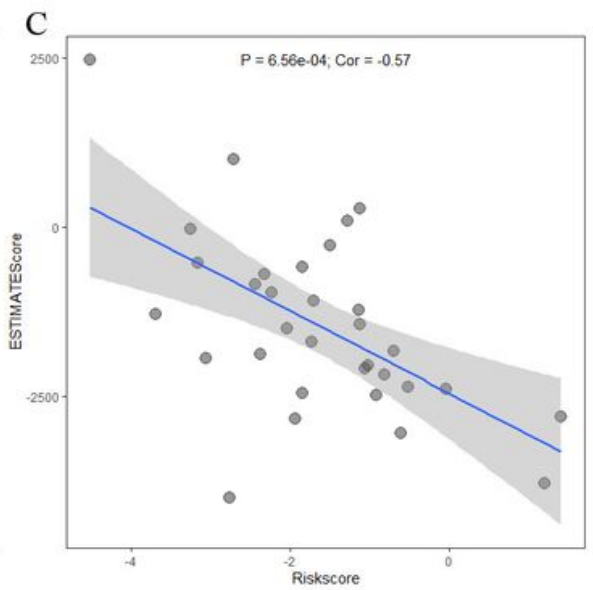

Figure 8

The correlation between the risk scores and tumor microenvironment scores. (A) Stromal score; (B) Immune score; (C) ESTIMATE score.
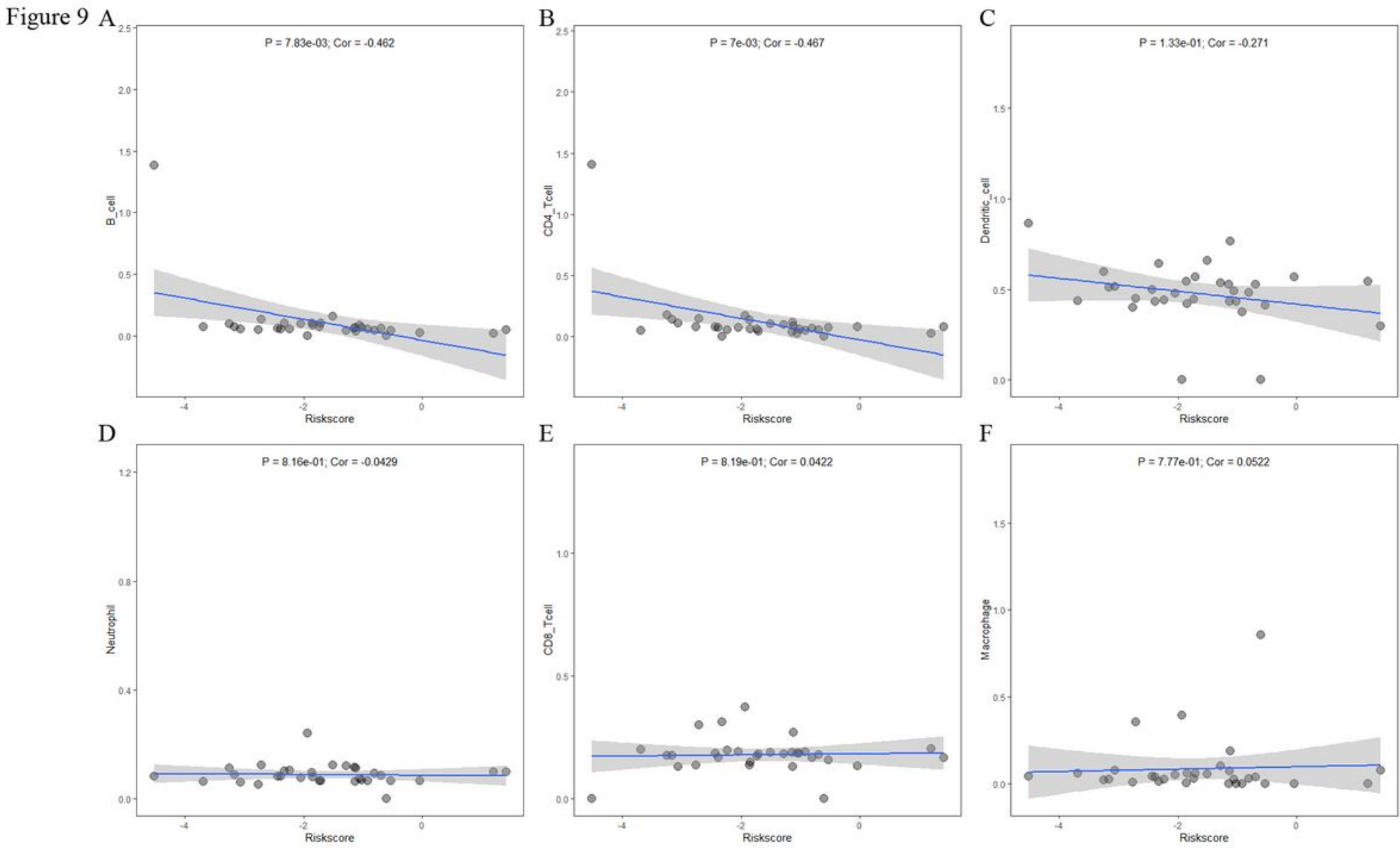

Figure 9

The correlation between the risk score and infiltration levels of six types of immune cells (A-F). (A) B cells, (B) CD4+ T cells, (C) Dendritic cell, (D) Neutrophil, (E) CD8+ T cells, (F) macrophage. 
Figure $10 \mathrm{~A}$ Boxplot of 22 immune infiltration cells

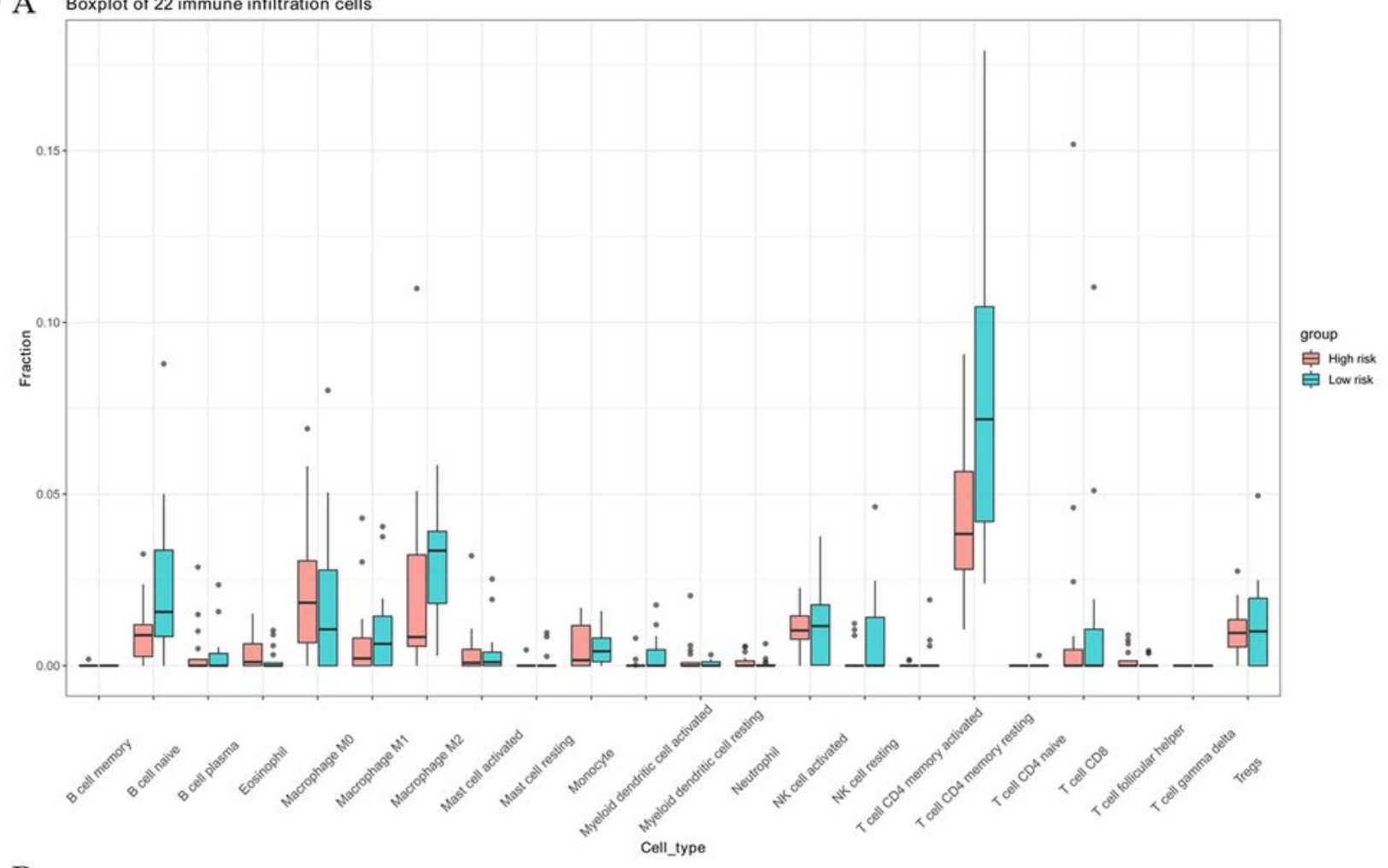

B

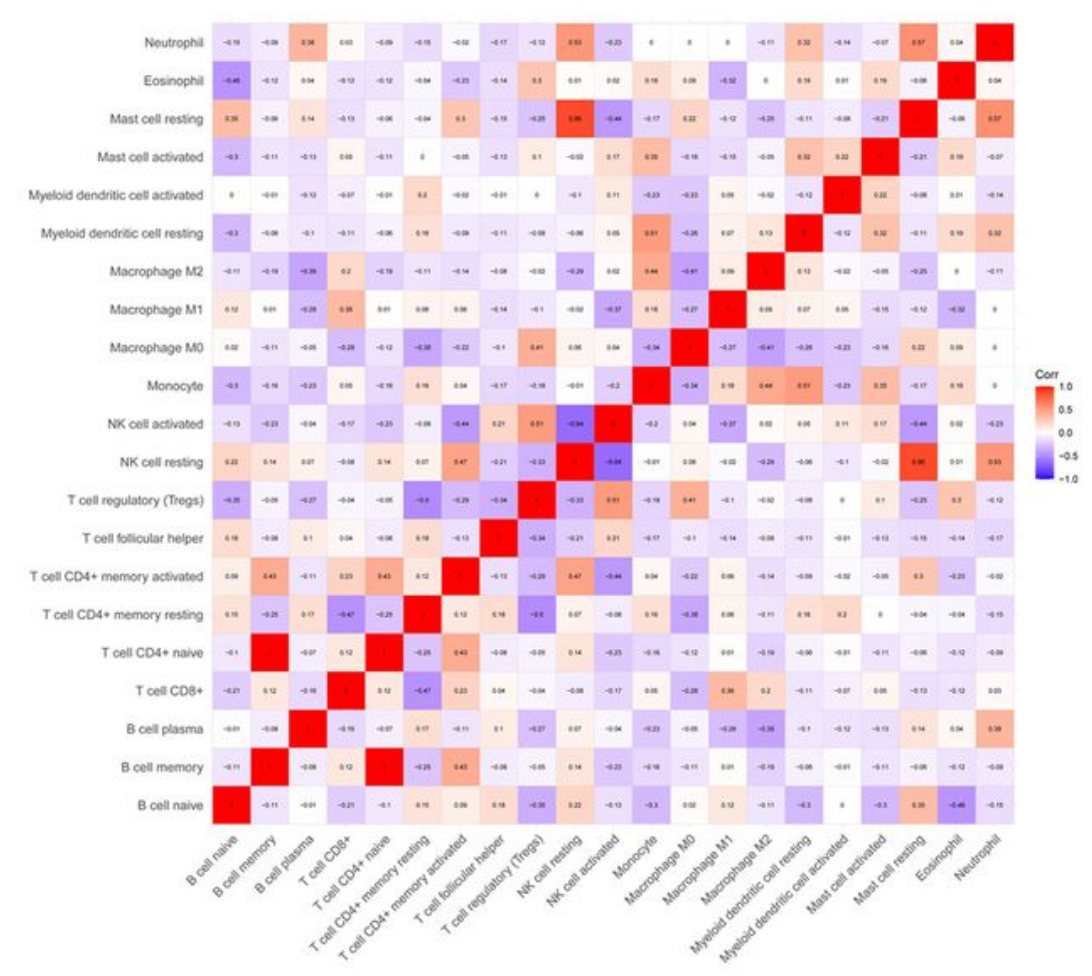

Figure 10

Difference and correlation analysis of tumor-infiltrating immune cells. (A) The difference of tumorinfiltrating immune cells between high- and low-risk score groups. (B) The correlation heatmap of 20 immune infiltration cells in CCA tumor samples. 
Figure $11 \mathrm{~A}$

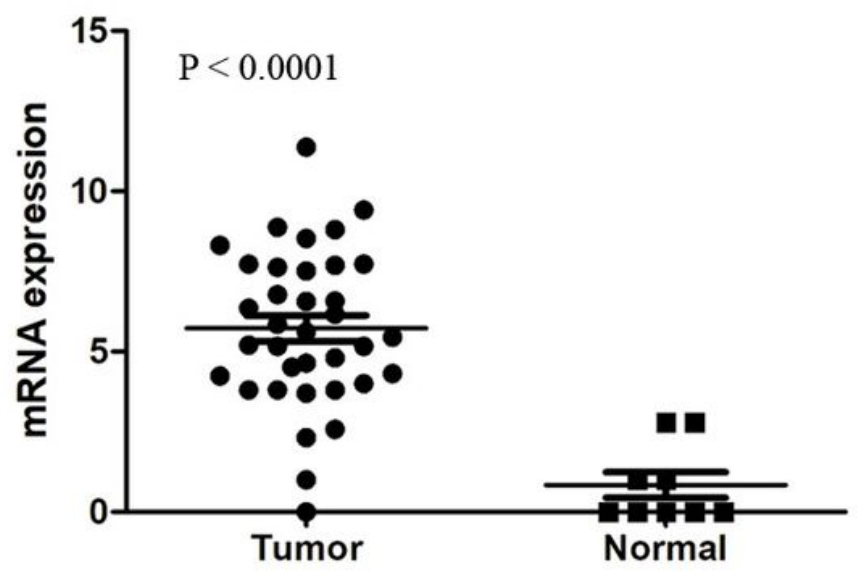

$\mathrm{C}$

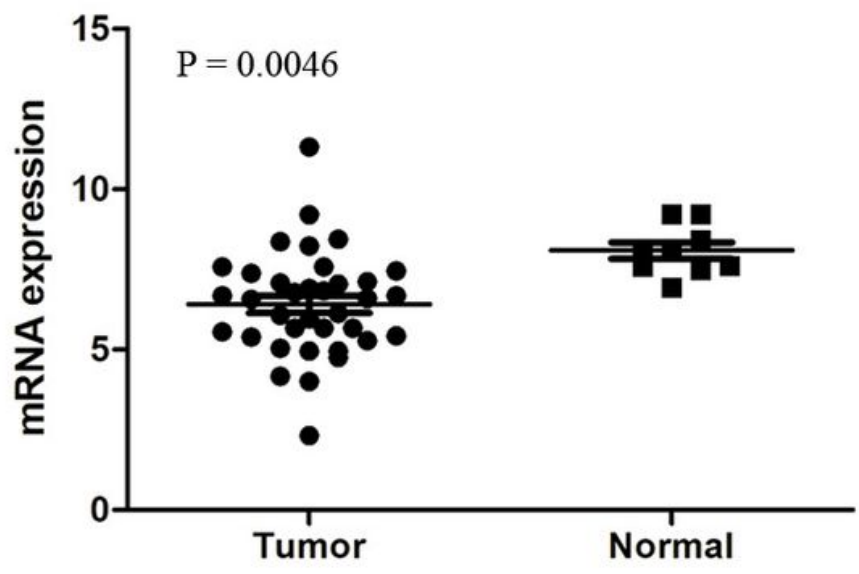

B

Survival curve $(p=8.25 e-03)$

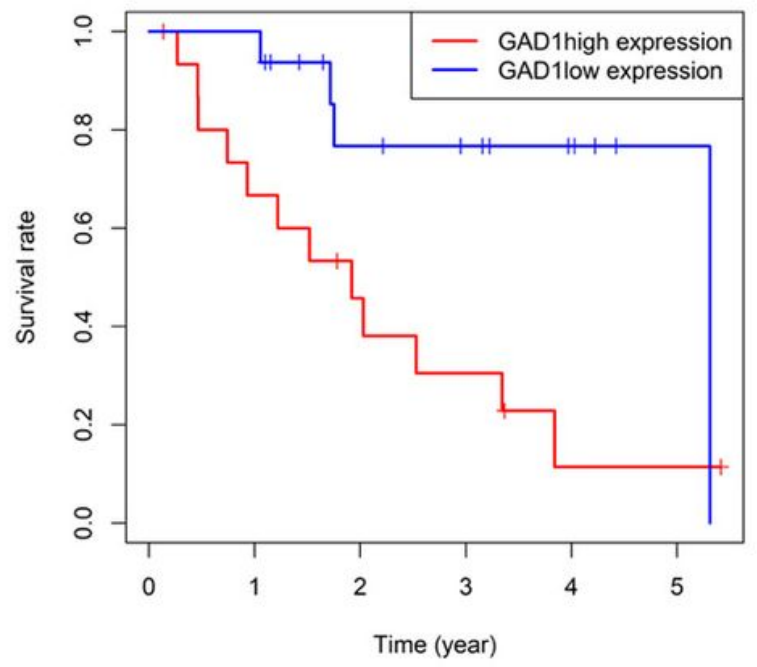

$\mathrm{D}$

Survival curve $(p=1.637 e-02)$

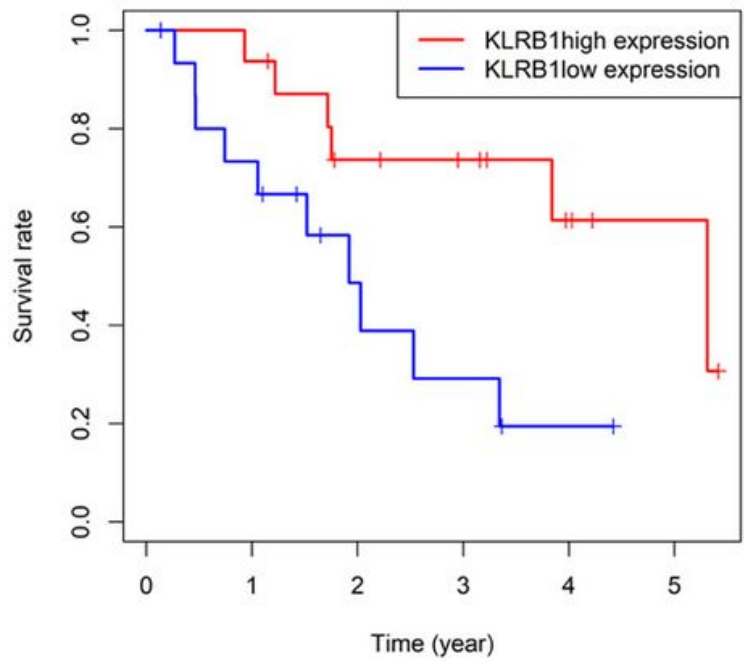

Figure 11

Expression and survival analysis of the two-gene signature in CCA patients. The mRNA expression levels of GAD1 (A) and KLRB1 (C) in TCGA CCA tumor tissue and normal tissue. (B, D) Kaplan-Meier survival analysis of the correlations between the expression levels of the two-gene signature and the OS.

\section{Supplementary Files}

This is a list of supplementary files associated with this preprint. Click to download.

- Additionalfile1.jpg

- FigureS1inMSbyCAOJX.jpg

- Table1.xlsx

- Table2.xlsx 
- Tables3.xlsx

- TableS4.xIsx

- Tables5.xIsx 